\title{
Spontaneous recovery after reversal and partial reinforcement
}

\author{
ROBERT A. RESCORLA \\ University of Pennsylvania, Philadelphia, Pennsylvania
}

\begin{abstract}
Six experiments used magazine approach in rat subjects to explore changes with time in responding for stimuli brought to a common moderate level of performance through acquisition or extinction. They found no evidence for increases with time in behavior during stimuli given simple acquisition. However, stimuli brought to that same level by reversal learning, repeated reversal, or partial reinforcement all showed increases in responding with time. These results su ggest that the decremental process established by nonreinforcement endures through subsequent reinforcement and is especially sensitive to the passage of time.
\end{abstract}

The phenomenon of spontaneous recovery, in which there is partial restoration of extinguished responding with the passage of time, is widely interpreted in terms of the differential temporal sensitivity of the processes responsible for acquisition and extinction. For instance, Pavlov (1927, pp. 48-67) was the first to suggest that acquisition occurred because reinforcement produced an excitatory association between the conditioned stimulus (CS) and the unconditioned stimulus (US) whereas extinction occurred because nonreinforcement superimposed an inhibitory process. He then accounted for spontaneous recovery after extinction in terms of the relatively greater fading of the inhibitory process with time. Although the inhibitory process involved in extinction has been described in a wide variety of ways, some involving learning and some only performance changes, a shared property has been this special sensitivity to the passage of time (see Rescorla, 2004a).

This paper examines the temporal sensitivity of the inhibitory process established by nonreinforcement in the context of two procedures that are somewhat more complex than simple extinction: repeated discrimination reversal and partial reinforcement. Both cases share with extinction the feature that the embedded nonreinforcement yields a reduced level of responding. But both also involve the continued delivery of a reinforcer.

The experiments are directed at two issues. First, how specific to an extinguished stimulus is the increase in responding observed with time? Put empirically, if two stimuli arrive at similar performance levels but differ in their history of nonreinforcement, will they show differential improvement with time? Put in a common theoretical language, if two stimuli have similar total associative strengths, but one achieves that level by a combination of substantial excitation from training and inhibition from extinction, whereas the other does so by having a moder- ate level of excitation, will spontaneous recovery be specific to the first? Experiments 1-3 examined this question for stimuli with different histories of reinforcement and nonreinforcement. Their results suggest that increases with time from a common performance level depend on a history of extinction. From a theoretical perspective, this suggests that the increases are a property of changes in the strength of the inhibitory component of a stimulus.

Second, is the inhibitory component produced by extinction at least partially preserved should that stimulus be reinforced? Experiments 2 and 3 examined that question by assessing spontaneous recovery for stimuli given reinforcement after initial extinction. Experiments 4-6 addressed it by assessing recovery for stimuli given concurrent training and extinction in the same sessions - that is, given partial reinforcement.

Experiments 1-3 explored changes in time for stimuli that were given initial discrimination training and then received 0,1 , or 5 reversals of that training. In each case, the final reversal training brought responding during the two discriminative stimuli to a common intermediate level. Then the stimuli were tested a week later, in order to provide the opportunity for responding to recover or to deteriorate from that common level. If the inhibition produced by nonreinforcement is especially sensitive to the passage of time, then stimuli with an extinction experience should show enhanced responding in the test, whereas stimuli having only a history of reinforcement should not. Experiments 4-6 explored the case in which the same stimulus received reinforcement and nonreinforcement intermixed on a daily basis. Responding was examined before and after a delay, with the intention of assessing the relative changes in the excitatory and inhibitory processes with the passage of time. For comparison, animals which had only a history of reinforcement were also examined. In Experiment 4 a conventional spontaneous recovery design

R.A. Rescorla, rescorla@cattell.psych.upenn.edu 
was used in which responding was examined during one stimulus immediately after treatment and during another stimulus one a week later. In Experiment 5 an alternative spontaneous recovery procedure was used in which two stimuli were tested in the same session which followed a delay of either 1 week or 1 hour after the most recent treatment. In Experiment 6 care was taken to insure similar levels of performance to a partially and continuously reinforced stimulus prior to giving them the opportunity for recovery.

All experiments employed a magazine approach preparation in rats. In this preparation a diffuse stimulus signals the arrival of a pellet at a food magazine. As the animal learns this relation it increasingly approaches and inserts its head into the magazine, a behavior that is measured as the conditioned response.

\section{EXPERIMENT 1}

The intention of this experiment was to compare the performance changes that occur with time during a stimulus that had undergone both acquisition and extinction with that during a stimulus that had only undergone acquisition, when both have attained the same response level prior to the delay. It is, of course, quite common to observe that responding will increase with time for a stimulus that has a history of both acquisition and extinction. But despite years of investigation there is little evidence that this increase is specific to a stimulus with such a history. As Robbins (1990) pointed out, it is in fact rare for investigators of spontaneous recovery to provide any comparison stimulus so as to pinpoint the increase with time as unique to a stimulus that was trained and then extinguished. Typically experiments simply compare responding to an extinguished stimulus before and after a time delay. To correct this, Robbins compared the changes in a well-extinguished stimulus with those in a stimulus that had only received nonreinforced exposure. He concluded from his observation of greater recovery in the former stimulus that spontaneous recovery is relatively specific to a trained and extinguished stimulus. However, the extremely low performance to both stimuli in Robbins's comparison leaves open the possibility that the two stimuli simply had different levels of overall associative strength from which they both recovered and which was exposed when the extinguished stimulus exceeded the response threshold.

In order to avoid this alternative, the present experiment compared a stimulus that received incomplete extinction to an intermediate level of responding with a stimulus that had achieved the same intermediate performance level by receiving only a moderate amount of simple acquisition. One can then ask whether performance to the two stimuli increases differentially from a common superthreshold level.

This procedure also permits one to ask about the possibility that a simply trained stimulus might show deterioration in its responding with time. Some interpretations of spontaneous recovery imply that there should be a symmetrical loss in responding to a stimulus that has had only acquisition in the same way that there is a gain in responding to an extinguished stimulus (e.g., Devenport, 1998; Estes, 1955). For instance, Estes (1955) suggested conceptualizing each stimulus as composed of elements whose presence on a trial varies randomly over time. When a previously conditioned stimulus is extinguished, then only those elements that occur during extinction trials are actually adversely affected. With the passage of time, some of those elements are increasingly replaced by other elements that were conditioned but were not present during extinction, yielding a test stimulus composed of a more balanced mixture of elements and hence capable of generating responding. As Estes noted, the same logic also applies to a simply trained stimulus, generating the prediction that its performance should deteriorate with time. Relatedly, Devenport (1998) has suggested that when an animal receives conflicting reinforcement and nonreinforcement experiences, its relative weighting of those at the time of test depends on their relative distance in the past. Immediately after extinction the nonreinforced experience is recent and acquisition more distant in the past. However, with the passage of time, their relative difference in time diminishes, resulting in an enhanced weighting for the learning experience and hence spontaneous recovery. That approach anticipates that if the two experiences were reversed in order, the complementary loss in behavior would occur.

Although there is little evidence for such loss in the time spans normally used to test spontaneous recovery, available experiments have commonly only examined stimuli that have been very well trained, raising the possibility that there is some undetected loss obscured by a ceiling effect. The present use of a moderate response level produced by only a modest amount of acquisition may offer a more promising opportunity to observe such loss.

\section{Method}

\section{Subjects and Apparatus}

The subjects were 16 male Sprague-Dawley rats about 90 days old. They were housed in individual cages and placed on a food deprivation regime that kept them at $80 \%$ of their ad lib body weight for the course of the experiment. They had free access to water in the home cage.

The apparatus consisted of eight operant chambers measuring $22.9 \times 20.3 \times 20.3 \mathrm{~cm}$, identical to those used in previous reports (e.g., Colwill \& Rescorla, 1985). The two end walls of each chamber were aluminum; the side walls and ceiling were clear Plexiglas. The floor of the chamber was composed of $0.48-\mathrm{cm}$ stainless-steel rods, spaced $1.9 \mathrm{~cm}$ apart, center to center. Each chamber had a recessed food magazine located behind a $4 \times 4 \mathrm{~cm}$ opening in the center of one end wall. An infra-red detector and emitter system was mounted on the side walls of the magazine, $1.5 \mathrm{~cm}$ behind the opening and $1.5 \mathrm{~cm}$ from the floor of the magazine, permitting automatic recording of head movements into the magazine.

Each chamber was enclosed in a sound- and light-resistant shell. Mounted on the inside wall of this shell were two speakers, one of which permitted the presentation of a white noise $(\mathrm{N})$ measuring approximately $76 \mathrm{~dB}$ re $20 \mu \mathrm{N} / \mathrm{m}^{2}$ against a background level of $62 \mathrm{~dB}$. Also mounted on that wall was a $6-\mathrm{W}$ bulb that could be illuminated to provide a light (L) stimulus during the otherwise dark session. A food dispenser containing 45-mg pellets (P. J. Noyes Co., Formula A) allowed delivery of pellets into the food magazine.

Experimental events were controlled and recorded automatically by relays and microprocessors located in an adjoining room. 


\section{Procedure}

Magazine training. On the 1 st day, the animals received a 20min magazine training session, during which 20 noncontingent deliveries of pellets were given, at time intervals variable around a mean of $1 \mathrm{~min}$.

Conditioning. On each of the next 6 days, all animals received eight $30-\mathrm{sec}$ presentations of both $\mathrm{L}$ and $\mathrm{N}$. For half the animals $\mathrm{L}$ was designated $\mathrm{A}$ and was followed by a pellet whereas $\mathrm{N}$ was designated $\mathrm{B}$ and nonreinforced; for the other half of the animals the roles of $\mathrm{L}$ and $\mathrm{N}$ were reversed. The mean intertrial interval (ITI) was $120 \mathrm{sec}$ measured between stimulus onsets. Stimuli were delivered in a quasirandom order with the restriction that no more than three successive trials could be of the same type.

Reversal. On each of the next 4 days, all animals received reversal training in which the previously reinforced A stimulus was extinguished and the previously B nonreinforced stimulus followed by a pellet. Each day contained 4 reinforced presentations of $B$ and 12 nonreinforced presentations of $A$. This differential trial frequency was used because prior work had suggested that the learning rates for acquisition are greater than those for extinction (e.g., Rescorla, 2002) and the goal was to achieve equal performance at an intermediate level.

Test. The animals spent the next 6 days in their home cages, maintained on a deprivation schedule, but without experimental sessions. On the next day all animals received a single test session. During this session $\mathrm{L}$ and $\mathrm{N}$ were each administered four times in counterbalanced random order without reinforcement.

\section{Results and Discussion}

Figure 1 shows responding over the course of acquisition, partial reversal, and test. That figure shows the mean responses per minute during each stimulus and during the 30 -sec stimulus free period prior to a trial. It is clear that the animals rapidly developed differential performance during acquisition. Responding climbed for the reinforced A stimulus but remained at the level of the prestimulus period during the nonreinforced B stimulus. With reversal training responding remained low in the prestimulus period but that during the stimulus periods converged.

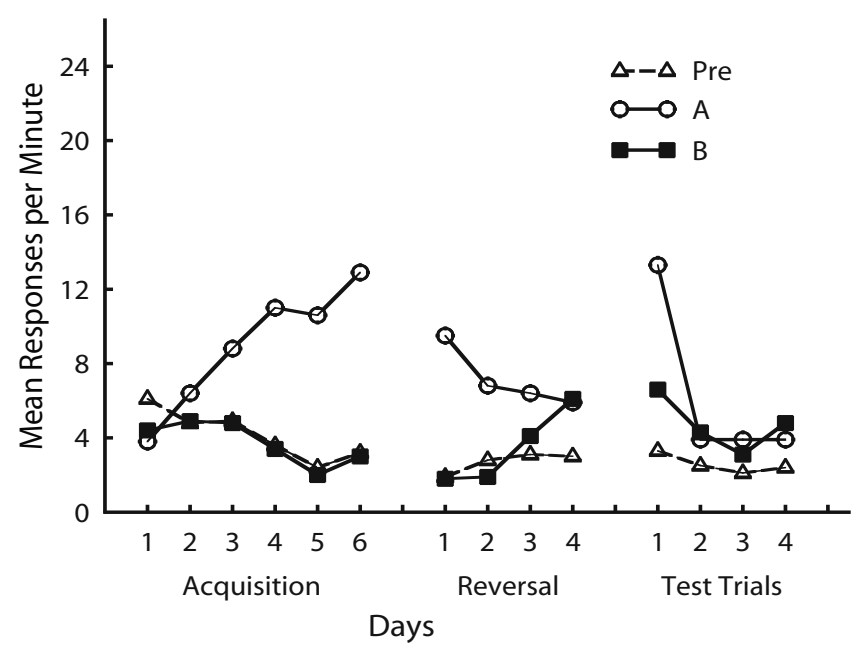

Figure 1. Mean responses per minute during acquisition, reversal, and delayed testing in Experiment 1. Responding is shown during the prestimulus period (Pre) and during each of two stim$u l i, A$, which was initially reinforced and then extinguished and $B$, which was originally presented without reinforcement and then conditioned to the same intermediate level.
The result was that by the final day of reversal there was no difference between the response rates during the two stimuli.

The results of most interest come from the test session given a week later. That session found differential performance on the first test trial of each type, followed by rapid return to a relatively lower level just above that exhibited during the prestimulus period. On the first test trial responding to the previously trained and extinguished A stimulus exceeded that it had shown at the end of reversal [Wilcoxon $T(13)=10, p<.01$ ] as well as that shown in the test during the simply trained B stimulus $[T(15)=11, p<.01]$. By contrast, responding to $\mathrm{B}$ was not different from that it had exhibited prior to the week's rest.

These results confirm the interpretation that is normally placed on spontaneous recovery, but seldom explicitly demonstrated, that the recovery is relatively specific to a stimulus that has undergone extinction. Consequently, these results show the sort of path-dependence that is implicit in most interpretations. Of equal importance, they show little evidence of loss in responding during the simply reinforced stimulus, even though it was tested after achieving the same response level as had the extinguished stimulus. These conclusions agree with those of prior investigators (e.g., Bouton \& Brooks, 1993) who found recovery in an extinguished stimulus but no loss in a trained stimulus, after more substantial reversal training to the point of each stimulus being near its new asymptotic level. The failure to find loss from both high and moderate response levels strengthens the suspicion of an asymmetry in the sensitivity of acquisition and extinction to the effects of time.

\section{EXPERIMENT 2}

This experiment is a systematic replication of Experiment 1 except that the matching of response level to the two stimuli followed an initial discrimination and a complete reversal learning. The result is that both stimuli had experienced some amount of both acquisition and extinction prior to the test for recovery. This yields another opportunity to observe differential recovery and/or loss but under circumstances in which the stimulus recently given reinforcement also has a history of extinction.

After initial $\mathrm{A}+/ \mathrm{B}-$ discrimination, and a complete $\mathrm{A}-/ \mathrm{B}+$ reversal, a partial $\mathrm{A}+/ \mathrm{B}-$ reversal brought responding during the stimuli to a common intermediate level prior to delayed testing. This experiment then continued the $\mathrm{A}+/ \mathrm{B}-$ reversal trials until both stimuli were asymptotic and then retested both stimuli after a week's delay. This allowed the conventional comparison of changes with time in responding to a recently reinforced and a recently extinguished stimulus from their terminal levels. Although this comparison between the two stimuli is less interesting because of their different response levels, this procedure may maximize the opportunity to see spontaneous recovery in the extinguished stimulus and provides a different performance level from which to observe loss in the reinforced stimulus. 


\section{Method}

\section{Subjects and Apparatus}

The subjects were 16 rats of the same type and maintained in the same manner as in Experiment 1. The apparatus was that of Experiment 1.

\section{Procedure}

The animals received initial magazine training in a manner identical to that used in Experiment 1. During conditioning, reversal, and testing the procedural details of Experiment 2 were those of Experiment 1 except where noted. Initial $A+/ B-$ discrimination learning extended over 10 days, as did the first A-/B + reversal. During each day, the animals received 8 reinforced and 8 nonreinforced trials, with $\mathrm{L}$ and $\mathrm{N}$ counterbalanced. On the next 2 days, the animals received a second reversal (i.e., a return to the initial A+/B- conditioning treatment), intended to bring the two stimuli to a common intermediate response level. As in the reversal of Experiment 1, each session contained four reinforced and 12 nonreinforced trials in order to attain a moderate level of performance to both stimuli at the point where they showed equal responding. The animals spent the next 6 days on deprivation in their home cages. On the following day, the animals were returned to the chambers for a test session identical to that used in Experiment 1.

On the next 8 days, the animals received additional A+/B - training in the same manner as that given on the two days prior to test. Then they received another 6 days in their home cages and the following day were tested for responding to both stimuli. This allowed us to see changes in the extinguished B stimulus from its low level of responding and in the reinforced A stimulus from its high level.

\section{Results and Discussion}

Figure 2 shows the course of acquisition, reversal, rereversal, and initial test in Experiment 2. As in Experiment 1 initial discrimination learning was straightforward. Initial reversal training was continued to the point at which responding to the now reinforced $B$ stimulus was high and that to the extinguished A stimulus was like that in the prestimulus period. Performance changed rapidly during the second reversal, so that similar levels of performance for $\mathrm{A}$ and $\mathrm{B}$ were attained on the second day.

After a week's rest, responding to the two stimuli differed dramatically, both on the initial test trial and throughout testing. Responding to the most recently extinguished B stimulus was markedly higher than both its own level prior to the rest $[T(16)=3, p<.01]$ and that to the most recently reinforced A stimulus during the test $[T(16)=21$, $p<.05]$. Moreover, the increase from the common level at the end of reversal was greater for the extinguished $\mathrm{B}$ stimulus $[T(16)=22, p<.05]$. However, unlike Experiment 1 , there was some evidence that there was also recovery in the most recently reinforced A stimulus. On the first test trial stimulus A showed a level reliably higher than it had prior to the rest $[T(14)=17, p<.05]$.

In most respects the results of this test are in agreement with those of Experiment 1. There was greater growth with time in the recently extinguished than in the recently reinforced stimulus. There was also no loss in responding to the most recently reinforced stimulus. One might have thought that the preceding of reinforcement with a prior history of extinction would have increased the chances of seeing regression. However, in this experiment the A stimulus actually showed some growth in responding, albeit less than did the recently extinguished B stimulus. Finding recovery with this sequence of treatments suggests that at least a moderate amount of retraining does not fully remove the ability of prior extinction to engender subsequent recovery. This partial preservation of the impact of nonreinforcement through a moderate period of training agrees with earlier work from this laboratory in a somewhat different procedure (Rescorla, 2001). That experiment found that an extinguished stimulus

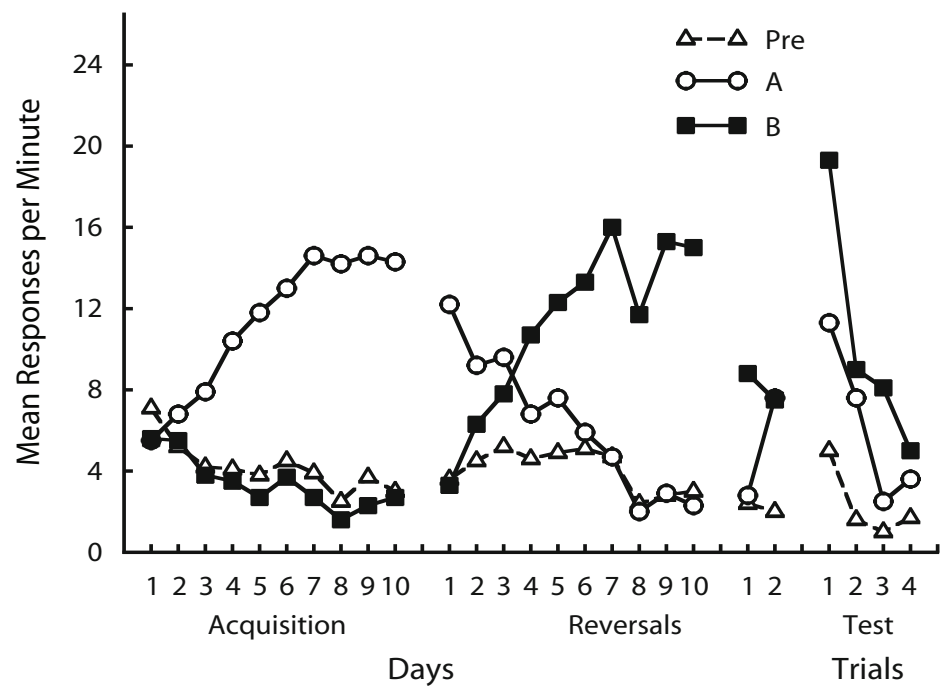

Figure 2. Mean responses per minute during acquisition, full reversal, partial reversal to a common level, and delayed testing in Experiment 2. Responding is shown during the prestimulus period (Pre) and during each of two stimuli, $A$, which was reinforced, then extinguished, and partially retrained as well as $B$, which was originally presented without reinforcement and then conditioned, and then partially extinguished. 


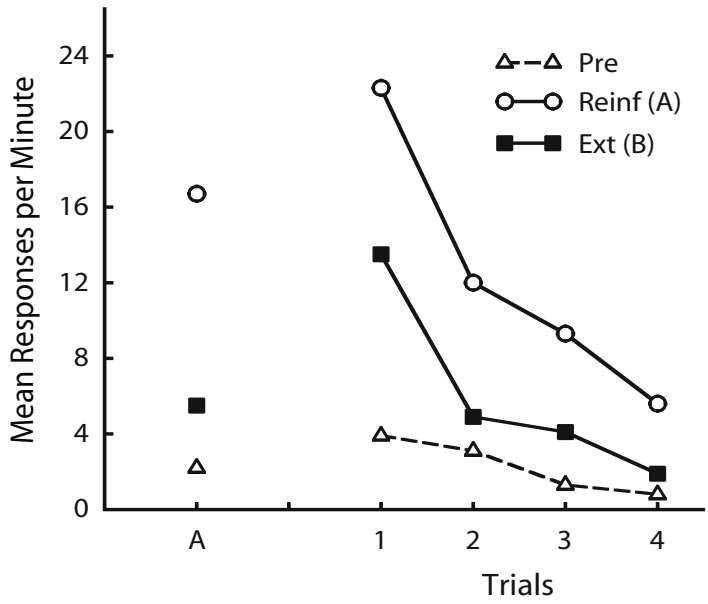

Figure 3. Mean responses per minute at the end of the full reversal and during a delayed test in Experiment 2. Responding is shown during the prestimulus period (Pre) as well as during the stimuli receiving reinforcement and extinction as their most recent treatment.

followed by a small amount of retraining continued to show spontaneous recovery with the passage time.

With a return to the $\mathrm{A}+/ \mathrm{B}-$ reversal training after the initial test, the animals rapidly achieved performance consistent with the reinforcement contingencies. The final levels of that performance, together with those of the test administered a week later are shown in Figure 3. In this figure, the stimuli are labeled by their most recent treatment, reinforcement (A) or extinction (B). It is clear that the passage of time resulted in substantial spontaneous recovery for the extinguished B stimulus. Responding to that stimulus was well above that observed at the end of its extinction $[T(16)=18, p<.01]$. Moreover, the passage of time had a similar incremental effect upon responding to the recently reinforced A stimulus. Far from showing regression over this period, A's performance also increased relative to its terminal reversal level $[T(16)=27, p<$ .05]. Although both stimuli showed enhanced responding, the discrimination between the stimuli was preserved over time $[T(16)=23.5, p<.05]$.

The increase in responding for the extinguished B stimulus represents the conventional observation of spontaneous recovery. The increase in responding to the recently reinforced A stimulus is somewhat more surprising. It suggests that performance increments can be observed even from the high level achieved at the end of training. Moreover, the history of nonreinforcement was sufficiently preserved through substantial subsequent reinforcement to permit that increment.

\section{EXPERIMENT 3}

This experiment is a further extension of Experiment 2 to include 5 full reversals of the reinforcement contingencies before bringing the stimuli to a common response level. The intention was to explore the generality of the findings of Experiment 2 under conditions in which the two stimuli both have substantial and more similar histories of reinforcement and nonreinforcement.

\section{Method}

\section{Subjects and Apparatus}

The subjects were 16 rats of the same type and maintained in the same manner as in Experiment 1. The apparatus was that of Experiment 1.

\section{Procedure}

The procedure was identical to that of Experiment 2 except where noted. Initial conditioning and each reversal was carried out over eight sessions. During conditioning and during the first three reversals, each session contained 8 presentations of each stimulus, one of which was reinforced. However, the sessions of the final two reversals each contained 4 presentations of the reinforced stimulus and 12 presentations of the nonreinforced stimulus. This was done in an attempt (which was not fully successful) to bring the level of responding during the nonreinforced stimulus down to the level of the prestimulus period in the limited number of sessions used. After five reversals, the animals were given a final rereversal for one session, which proved to be adequate for attaining similar response levels. By the end of this repeated reversal, the animals had received a total of $164 \mathrm{~A}+, 224 \mathrm{~A}-, 160 \mathrm{~B}+$, and $236 \mathrm{~B}-$ trials. After spending 6 days on deprivation in their home cages, on the next day all animals were tested in the manner of previous experiments.

\section{Results and Discussion}

As shown in Figure 4, the results of this experiment were in agreement with those of Experiment 2. Initial rapid discrimination learning was successfully reversed and rereversed multiple times, although in later reversals overall responding increased and that during the nonreinforced stimulus remained above that in the prestimulus period. In the test after a week's delay, the most recently nonreinforced B stimulus showed a dramatic recovery of responding. That recovery proved reliable relative both to its own level before the rest $[T(16)=0, p<.01]$ and to the

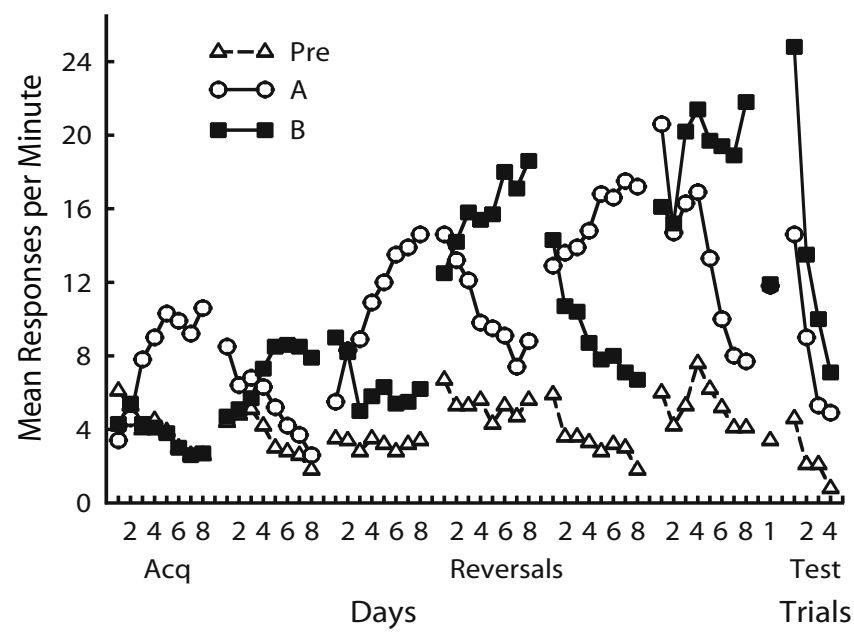

Figure 4. Mean responses per minute during acquisition, full reversal, partial reversal to a common level, and delayed testing in Experiment 3. Responding is shown during the prestimulus period (Pre) and during each of two stimuli, $A$ and $B$, each of which received repeated reversal prior to being brought to a common response level before testing. 
level of the most recently reinforced A stimulus during the test $[T(16)=0, p<.01]$. In addition, the increase from the common level at the end of the reversal was greater in the recently B extinguished stimulus $[T(16)=11.5, p<$ $.01]$. Moreover, as in Experiment 2 the most recently reinforced A stimulus also showed an enhanced responding $[T(16)=30, p<.05]$, rather than any sign of loss.

These results confirm the conclusions of Experiment 2 that there is greater spontaneous recovery in the stimulus most recently extinguished. But they also agree in finding some sign of increase in a stimulus with a history of extinction despite the fact that its most recent treatment is reinforcement. Again, these results suggest that there not only is no regression but that the recovery-inducing impact of extinction survives some level of reinforcement.

In some ways it is remarkable that there is differential recovery in the two stimuli with such similar histories. Both stimuli had been trained and extinguished multiple times and then brought to a common response level. Their primary difference was in their most recent experience. Apparently the recency of this treatment is a partial determinant of the magnitude of recovery.

\section{EXPERIMENT 4}

The most extreme example of repeated reversal learning is simple intermixed reinforcement and nonreinforcement of the same stimulus. The success of the previous experiments in finding recovery with time after repeated reversals suggests that one might see a similar recovery in the case of such partial reinforcement. If nonreinforcement induces a process that permits recovery and a moderate amount of subsequent reinforcement does not fully undermine that process, then one would expect that imposing a period of rest after partial reinforcement would result in enhanced responding. Such an observation would provide strong confirmation for the differential growth with time in acquisition and extinction processes from a common performance level.

For this reason Experiments 4-6 both explored recovery after partial reinforcement. Experiment 4 used a relatively standard within-subjects spontaneous recovery design. In that experiment two stimuli were given partial reinforcement and then one stimulus was tested immediately after training and the other was tested after a week's delay. As Rescorla (2004a) noted, this kind of design is an improvement over the commonly used design adopted in Experiments $1-3$, which requires repeated testing of the same stimulus. However, Rescorla also noted that even this test procedure has the flaw that comparison must be made of responding at two quite different points in time, when the animals might be in quite different states. Therefore, Experiment 5 examined recovery after partial reinforcement using an alternative design in which responding was assessed for two stimuli during the same test session under circumstances in which one stimulus had recently been treated whereas the other had been similarly treated more distantly in the past. This allows one to compare the stimuli in the same session when they have had differential opportunities for recovery with time. In both experiments companion groups that re- ceived continuous reinforcement were also run. Although it was expected that these groups would show greater performance, they provided some opportunity to obtain evidence for the specificity of any recovery to stimuli with a history of partial reinforcement. Experiment 6 compared changes in time after partial and continuous reinforcement under conditions designed to correct this.

\section{Method}

\section{Subjects and Apparatus}

The subjects were 32 rats of the same type and maintained in the same manner as in Experiment 1. The apparatus was that of Experiment 1.

\section{Procedure}

Conditioning. The animals received initial magazine training in a manner identical to that used in Experiment 1. On each of the next 8 days, the 16 animals in the partial group received eight 30 sec reinforced presentations each of $\mathrm{L}$ and $\mathrm{N}$, intermixed with eight nonreinforced presentations of each stimulus. The 16 animals in the continuous group received the same treatment except that the eight nonreinforced presentations of each stimulus were deleted. Stimuli were delivered in a quasirandom order with the restriction that no more than three successive trials could be of the same type. For both sets of animals the mean ITI was $120 \mathrm{sec}$.

Testing. Approximately $1 \mathrm{~h}$ after the end of the last conditioning session, the animals were returned to the chambers for an initial test. During this test each animal received four nonreinforced presentations of either $\mathrm{L}$ or $\mathrm{N}$, half of the animals within each group receiving $L$ and half receiving $N$. The animals spent the next 6 days without treatment in the home cages, but maintained on their deprivation schedule. On the following day, the animals were returned to the chambers for a second test. This test was identical to the first except that it was performed with the alternative stimulus.

\section{Results and Discussion}

Training proceeded smoothly with both partial and continuous reinforcement. With neither treatment was there a reliable difference in responding between $\mathrm{L}$ and $\mathrm{N}$. Not surprisingly, there was overall greater responding with continuous reinforcement.

Figure 5 shows the terminal levels of responding for both conditions, separated according to whether the stimulus would be tested immediately or after a week's delay. That figure also shows the results of the two test sessions. Since there were no differences in prestimulus responding those results have been combined for the two sessions for simplicity of reporting and consistency with the way they are presented in Figure 6.

It is clear that for the partially reinforced animals both stimuli continued to elevate responding relative to the prestimulus period. However, the stimulus that was tested after a delay showed greater responding than did the stimulus tested immediately $[T(15)=25, p<.05]$. Not surprisingly, the stimulus tested immediately produced a level of responding similar to that it had at the end of conditioning $[T(15)=52$, n.s. $]$. The greater responding after a delay suggests that in the partially reinforced animals there was a "recovery" of responding like that observed in prior experiments.

The overall level of responding in the continuously reinforced animals was higher, although well below a response ceiling. In those animals there was no sign that imposing 
a delayed period led to enhanced responding, either on the first trial nor on later trials, during which the response level was more similar to that of the partially reinforced animals. This failure to obtain response enhancement with continuous reinforcement is consistent with the results of Experiment 1 as well as with earlier results from this laboratory with similar procedures (e.g., Rescorla, 1997).

The differences in overall response levels make direct comparisons between the partial and continuous animals hazardous. Nevertheless, the difference in responding between the immediate and delayed test was reliably greater for the partially reinforced animals than for the continuously reinforced animals [Mann-Whitney $U(16,16)=73$, $p<.05]$.

\section{EXPERIMENT 5}

As noted above, the standard procedure for measuring recovery with time has certain drawbacks in terms of requiring comparisons across sessions given at quite different points in time. One can develop alternative procedures in which the training is staggered such that stimuli differing in the time since their treatment are nevertheless tested in the same session. In discussing these alternatives, Rescorla (2004a) recommended using both kinds of procedures in any assessment of recovery. Consequently, this experiment used a version of that alternative procedure.

In this experiment, animals are first given most of their initial conditioning with both $\mathrm{L}$ and $\mathrm{N}$ in the same sessions. Then each animal received two additional sessions with one of the stimuli and, after a delay, two additional sessions with the other stimulus. Then the animal was immediately tested with both stimuli in the same session. This procedure results in differential delays between the end of treatment and test for two stimuli despite their being tested in a common session. One stimulus is tested immediately after its training and one with a delay after its

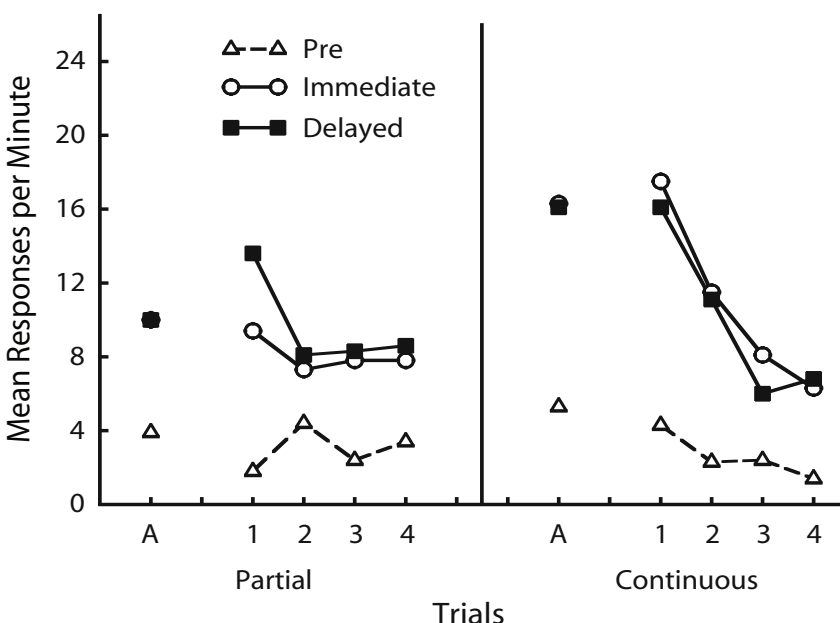

Figure 5. Mean responses per minute at the end of acquisition (A) and over the course of testing for animals trained with partial or continuous reinforcement in Experiment 4. One stimulus was tested immediately after training and one was tested after a week's delay.

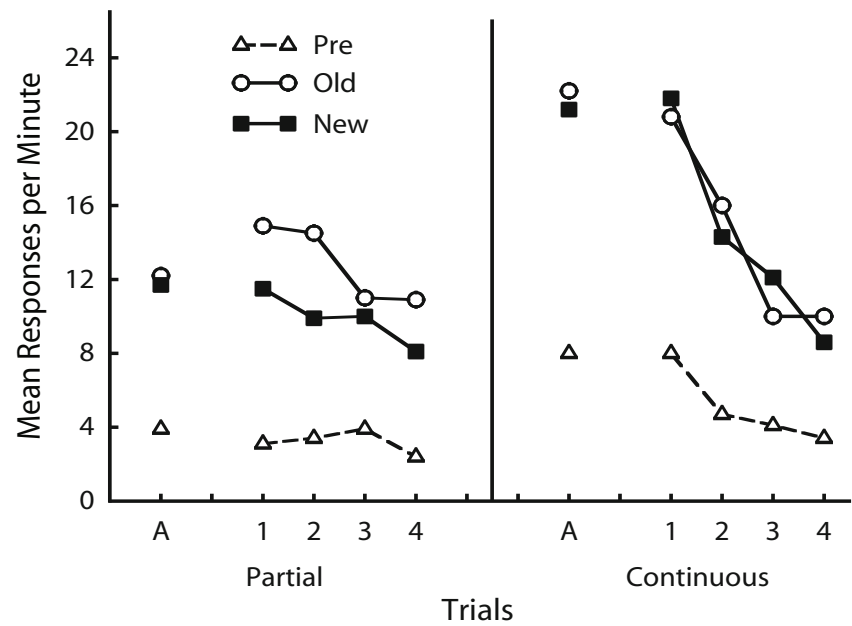

Figure 6. Mean responses per minute at the end of acquisition (A) and over the course of testing for animals trained with partial or continuous reinforcement in Experiment 5. One stimulus (New) was recently trained whereas the other (Old) had received its last training further in the past.

training. Such a procedure has been frequently employed successfully in this laboratory in the investigation of spontaneous recovery.

\section{Method}

\section{Subjects and Apparatus}

The subjects were 32 rats of the same type and maintained in the same manner as in Experiment 1. The apparatus was that of Experiment 1.

\section{Procedure}

The procedure was intended as a companion to that used in Experiment 4. After initial magazine training, all animals received six sessions of conditioning identical to those used in Experiment 4. Half the animals had eight reinforced presentations of $\mathrm{L}$ and $\mathrm{N}$ in each session; the other half of the animals had an additional eight nonreinforced presentations of each stimulus. On each of the next 2 days, all animals received additional training on one of the two stimuli, either $\mathrm{L}$ or $\mathrm{N}$. The partial animals received eight reinforced and eight nonreinforced presentations of the stimulus each day whereas the continuously reinforced animals received eight reinforced presentations each day. After a rest period of 5 days without treatment, the animals received two additional sessions in which they received the same treatments with the alternative stimulus, eight reinforced each day for the continuous animals and eight reinforced mixed with eight nonreinforced each day for the partial animals. Approximately $1 \mathrm{~h}$ after the last session all animals received a test session which contained four nonreinforced presentations each of $\mathrm{L}$ and N. Stimulus assignment was fully counterbalanced throughout the experiment. Half of each set of animals received initial training with $\mathrm{L}$ followed by later training with $\mathrm{N}$ and half received the converse. During the test session the stimuli were presented in balanced order, with the first stimulus being that most recently trained in half the animals.

\section{Results and Discussion}

The results of this experiment were highly similar to those of Experiment 4. Continuous reinforcement produced greater responding than did partial reinforcement. Figure 6 shows responding during the final session of training and over the test for both conditions. As in earlier 
applications of this procedure, there were no reliable differences between the stimuli given the first or the second treatment, so those results have been combined for the final acquisition session shown in Figure 6. As in Experiment 4, the partially reinforced animals showed greater responding to the stimulus with delayed testing compared to that with immediate testing $[T(14)=16, p<.01]$. Similarly, there was overall greater responding in the continuously reinforced animals, but no sign of a greater responding in the stimulus tested after a delay. As in Experiment 4, despite the differences in response level, the greater responding to the stimulus trained more distantly in the past than to the more recently trained stimulus proved larger for the partial animals $[U(16,16)=74, p<.05]$.

It should be noted that although both partially reinforced stimuli received the bulk of their training during the same sessions, their final two sessions were separated by 5 days in order that the delay between final training and test might permit recovery for one stimulus but not the other. The result of this is that there was a 5-day delay prior to the final two training sessions for one stimulus. Comparing the initial trial of the first of these final two sessions for each stimulus the therefore provides the opportunity to make the conventional comparison for spontaneous recovery used in Experiment 4. Although the stimuli did not differ in responding for these final sessions as a whole, they did show a difference on the initial trial. On that trial the mean response rates were 9.8 and 12.6 for the stimulus with a one-day and the stimulus with a 5 -day delay $[T(16)=23, p<.05]$. No similar difference was observed for the continuously reinforced stimulus.

These results confirm those of Experiment 4, using a different procedure for testing recovery, that partially reinforced stimuli show a growth in responding with a delay. That observation is consistent with the view that the decremental effects of nonreinforcement dissipate with time more rapidly than do the incremental effects of reinforcement.

\section{EXPERIMENT 6}

The designs used in Experiments 4 and 5 found evidence for response increases with time in stimuli given conditioning with partial reinforcement. Although they did not find comparable increases with continuously reinforced stimuli, the differences in responding at the end of acquisition make detailed comparisons suspect. The intention of Experiment 6 was to use a conditioning procedure that matches the levels of performance at the end of acquisition for stimuli given partial and continuous reinforcement so that comparisons like those used in Experiments 1-3 can be made. In order to accomplish this, animals were given initial conditioning with a partially reinforced stimulus until performance was stable. At that point a continuously reinforced stimulus was given just sufficient conditioning to bring its performance to the level of the partially reinforced stimulus. Both stimuli were then tested from that common performance level after a week's rest.

\section{Method}

\section{Subjects and Apparatus}

The subjects were 16 rats of the same type and maintained in the same manner as in previous experiments. The apparatus was that of Experiment 1.

\section{Procedure}

After initial magazine training like that used in prior experiments, all animals received 10 days of conditioning. Each session contained sixteen 30 -sec presentations of a stimulus, spaced with a mean ITI of 120 seconds, reinforced on a $50 \%$ schedule. For half of the animals the stimulus was the noise and for half it was the light.

On the next session, each animal received eight intermixed presentations each of the light and of the noise. The new stimulus was followed by a pellet on each trial; the previously conditioned stimulus was reinforced on half of its occurrences. Prior work in our laboratory had indicated that this training would be sufficient to yield similar response levels on the two stimuli by the end of the single session.

The animals spent the next 6 days on a deprivation schedule in their home cages. On the following day, all animals received a single test session. During this session, each stimulus was presented and nonreinforced four times, in a manner that balanced their order within session and with regard to prior treatments. The issue of interest was the degree to which the two stimuli might show different changes in response rate with the passage of time.

\section{Results and Discussion}

Figure 7 shows responding during the stimuli and in the prestimulus period throughout the course of the experiment. The left hand side of the figure shows the daily responding during the conditioning of the partially reinforced stimulus. Responding grew to an intermediate level for the partially reinforced stimulus over the course of the 10 conditioning days. The middle panel of the figure shows responding to the two stimuli, plotted in blocks of 2 trials, during the single day in which the $100 \%$ stimulus was added. It is clear that responding was initially low to the continuously reinforced stimulus but rapidly grew during that session to a level just slightly above that of the $50 \%$ stimulus. On the last trial block, the was not a reliable difference in responding to the two stimuli.

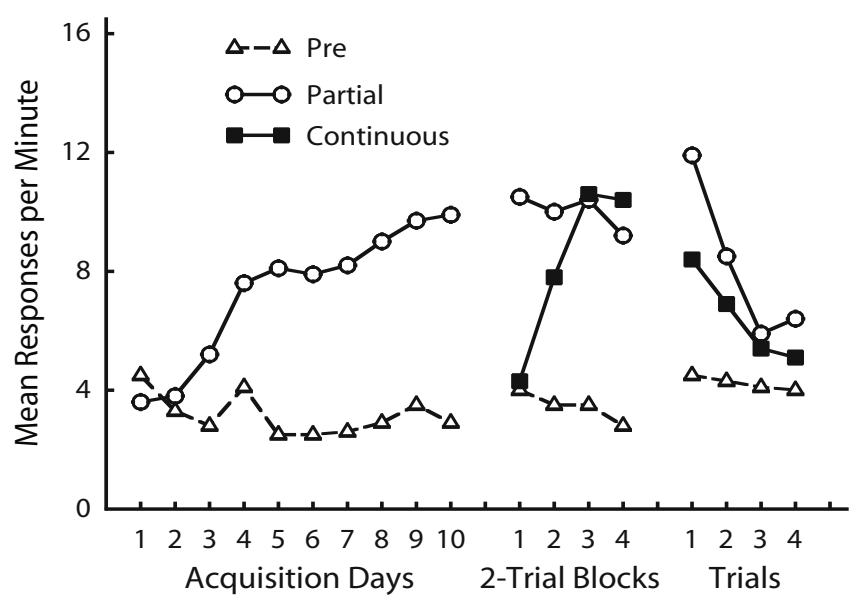

Figure 7. Mean responses per minute over the course of acquisition for the partially reinforced stimulus, acquisition for the continuously reinforced stimulus, and testing. 
The results of most interest, from the final test given a week later, are shown to the right in Figure 7, plotted on a trial by trial basis. During this test, responding to both stimuli declined, but was initially greater to the partially reinforced stimulus. On the first test trial, the partially reinforced stimulus produced reliably greater responding than did the continuously reinforced stimulus $[T(12)=$ $14, p<.05]$. Comparison between responding on the final acquisition trials and the initial test trials confirms this. Between the end of acquisition and the first test trial, the partially reinforced stimulus showed a reliable increase $[T(14)=16.5, p<.05]$, whereas the continuously reinforced stimulus showed a nonreliable decline.

The results of this experiment strengthen the impression gained from Experiments 4 and 5 that there is a growth in responding with time after partial reinforcement but not after continuous reinforcement. However, it is clear that none of the experiments by itself is immune to alternative interpretation. Although the present experiment did solve the difficulty of having different terminal response levels, it did so at the expense of introducing other differences between the two stimuli. For instance, the conditioning of the partial stimulus was conducted over multiple days and the acquisition curve was gradual whereas the conditioning of the continuous stimulus was conducted in one day and the curve very steep. Nevertheless the evidence suggests that using a variety of procedures there is a growth in responding with time after partial reinforcement that is not matched following continuous reinforcement.

It is worth noting that the continuously reinforced stimulus acquired responding very rapidly in this experiment. Although responding was at the level of the prestimulus period on the initial two trials, the stimulus evoked substantial responding by trials 5 and 6 . Although the basis of this rapid learning is not clear, it agrees with earlier work in this laboratory as well as eye blink studies reported by Kehoe (e.g., Kehoe, Morrow, \& Holt, 1984).

\section{GENERAL DISCUSSION}

These experiments found evidence that the decremental process involved in nonreinforcement has a special sensitivity to the passage of time. After simple extinction, reversal learning, repeated reversal learning, and partial reinforcement, a recently nonreinforced stimulus shows growth in responding from an intermediate level of performance that is markedly greater than that shown by control stimuli having the same level of performance.

In none of these experiments did a simply reinforced stimulus show reliable evidence for either a growth or a loss in responding with time. The absence of a growth in responding from an intermediate level of performance provides a much needed comparison for the changes exhibited by an extinguished stimulus. The absence of a loss in responding for a simply trained stimulus seems inconsistent with any view of spontaneous recovery from extinction that sees it as solely attributable to a restoration of the balance between the most recent learning experience and that which preceded it with the passage of time (e.g., Devenport, 1998; Estes, 1955) Although such views may provide a useful framework for interpreting recovery from extinction, they also predict a symmetrical regression after acquisition.

However, Experiments 2 and 3 did show some evidence that a recently reinforced stimulus with a history of extinction can show an increase in responding with time. This increase apparently represents the continued action of prior nonreinforcement despite intervening reinforcement. It confirms the inference from prior experiments (e.g., Rescorla, 2001) that reinforcement does not remove a history of nonreinforcement, paralleling the well-accepted view that nonreinforcement does not remove the history of reinforcement. Presumably it is the ability to preserve this ambiguous history that also allows responding to increase after partial reinforcement in Experiments 4, 5, and 6.

The special sensitivity to the passage of time of the inhibition established by nonreinforcement has several possible underlying mechanisms. Historically it has been popular to speculate that inhibition simply fades more rapidly with time than does excitation. However, Bouton (e.g., Bouton \& Brooks, 1993) has suggested a somewhat different alternative according to which inhibition is especially likely to come under the control of temporal stimuli. On this view, the passage of time produces not a deterioration in the strength of the learned inhibition but rather a relative absence during the test conditions of the stimulus that controls that inhibition. Given that temporal stimuli are presumed to change monotonically with time, these two alternatives may prove difficult to disentangle empirically. However, Brooks and Bouton (1993) have found evidence that the inhibition involved in nonreinforcement can be brought under the control of both contextual and discrete stimuli, a result consistent this view. Moreover, Rescorla (2004b) has reported that spontaneous recovery from extinction is sensitive not simply to the absolute passage of time but rather to the relative temporal interval distances of acquisition and extinction from the test. The most natural interpretation of a fading of inhibition position is that the change should depend on the actual time that has passed. By contrast, it is relatively easy to imagine that the judgment of the similarity between the stimuli present in extinction and test could be made relatively.

It is worth noting that although the present experiments successfully match the prerecovery levels of performance for various stimuli, it is unlikely that they match the strengths of the underlying excitatory and inhibitory processes engaged by acquisition and extinction, respectively. Indeed, the very fact that the animals show some performance to a stimulus is evidence that the excitatory processes of acquisition are currently stronger than the inhibitory processes of extinction. Consequently, one might view these results as suggesting that the currently weaker of the two processes (in this case, inhibition) is more vulnerable to the passage of time. This would naturally account for the growth in responding after simple extinction and after partial reinforcement, as well as the lack of growth for a stimulus receiving simple acquisition (which has only the excitatory process). It is less clear how it can account for the differential recovery of the recently reinforced and recently extinguished stimuli after repeated re- 
versal. Their similar performance levels suggests that they have similar total associative strengths, reflecting similar differences between the strengths of the excitatory and inhibitory processes, yet they recover differently. Those results suggest that the recovery is at least partly determined by the recency of a particular experience. But apparently that recency has an asymmetrical impact such that there is not simply a reversion to the behavior engendered by the just previous conditioning contingencies, but rather is differential for excitation and inhibition.

It should also be kept in mind that most of the experiments reported here employed the conventional procedure for studying spontaneous recovery, in which the same stimulus is tested twice, immediately and after a delay. Only in the case of partial reinforcement was an alternative, and arguably superior, testing procedure used.

The comparison of two stimuli, one trained and one extinguished, that evoke similar performance levels, has also been made using various other experimental manipulations. Recently, Rescorla (2003) has found evidence that these stimuli differ in their sensitivity to new reinforcement contingencies. He reported that a trained and then extinguished stimulus was more sensitive both to the augmenting effects of reinforcement and to the depressing effects of nonreinforcement. One might take the apparent earlier initial point of performance equality with each successive reversal in Experiment 3 as consistent with that observation. Similarly, Swartzentruber and Rescorla (1994) found evidence that despite evoking similar performance levels when presented separately, a simply trained stimulus and a trained but then extinguished stimulus were differentially sensitive to the superimposition of modulators. The trained and then extinguished stimulus was both more easily augmented by a conditioned facilitator and more easily depressed by a conditioned inhibitor. However, despite these differential sensitivities, Rescorla (2002) has found that such stimuli are similar in the level of performance that they evoke in a summation paradigm in which they are presented in compound with a moderate excitor. This data pattern suggests that a summation test reflects the net associative strength of a stimulus whereas various modulatory procedures are sensitive to the degree to which that net strength is achieved by counterbalancing excitatory and inhibitory processes.

Perhaps the most interesting observation of these experiments is the growth in time in responding to partially reinforced stimuli. This observation suggests that the intermixing of reinforcement and nonreinforcement for the same stimulus at least partially preserves the properties of the component processes. That is consistent with classical conditioning/extinction theories of such treatments and of discrimination learning (e.g., Spence, 1936). Of course, it does not preclude the emergence of some additional process produced by the intermixing of treatments, such as the partial reinforcement extinction effect. But it may sug- gest that that effect should be especially prominent in the early trials of multiday extinction treatments during which spontaneous recovery could be observed.

In any case, the present experiments confirm the suspicion based on earlier observations that the decremental process produced by extinction is especially sensitive to the passage of time.

\section{AUTHOR NOTE}

This research was supported by National Institutes of Health Grant R01 MH67848. Correspondence concerning this article should be addressed to R. A. Rescorla, Department of Psychology, University of Pennsylvania, 3720 Walnut Street, Philadelphia, PA 19104 (e-mail: rescorla@cattell.psych.upenn.edu).

\section{REFERENCES}

Bouton, M. E., \& Brooks, D. C. (1993). Time and context effects on performance in a Pavlovian discrimination reversal. Journal of Experimental Psychology: Animal Behavior Processes, 19, 165-179.

Brooks, D. C., \& Bouton, M. E. (1993). A retrieval cue for extinction attenuates spontaneous recovery. Journal of Experimental Psychology: Animal Behavior Processes, 19, 77-89.

Colwill, R. M., \& Rescorla, R. A. (1985). Post-conditioning devaluation of a reinforcer affects instrumental responding. Journal of Experimental Psychology: Animal Behavior Processes, 11, 120-132.

DEVENPORT, L. D. (1998). Spontaneous recovery without interference: Why remembering is adaptive. Animal Learning \& Behavior, 26, 172-181.

Estes, W. K. (1955). Statistical theory of spontaneous recovery and regression. Psychological Review, 62, 145-154.

Kehoe, E. J., Morrow, L. D., \& Holt, P. E. (1984). General transfer across sensory modalities survives reductions in the original conditioned reflex in the rabbit. Animal Learning \& Behavior, 12, 129-136.

Pavlov, I. P. (1927). Conditioned reflexes (G. B. Anrep, Trans.). London: Oxford University Press.

Rescorla, R. A. (1997). Spontaneous recovery after Pavlovian conditioning with multiple outcomes. Animal Learning \& Behavior, 25, 99-107.

ResCorla, R. A. (2001). Retraining of extinguished Pavlovian stimuli. Journal of Experimental Psychology: Animal Behavior Processes, 27, 115-124.

RESCORLA, R. A. (2002). Comparison of the rates of associative change during acquisition and extinction. Journal of Experimental Psychology: Animal Behavior Processes, 28, 406-415.

RESCORLA, R. A. (2003). More rapid associative change with retraining than with initial training. Journal of Experimental Psychology: Animal Behavior Processes, 29, 251-260.

Rescorla, R. A. (2004a). Spontaneous recovery. Learning \& Memory, 11, 501-509.

Rescorla, R. A. (2004b). Spontaneous recovery varies inversely with the training-extinction interval. Learning \& Behavior, 32, 401-408.

RoBbins, S. J. (1990). Mechanisms underlying spontaneous recovery in autoshaping. Journal of Experimental Psychology: Animal Behavior Processes, 16, 235-249.

SPENCE, K. W. (1936). The nature of discrimination learning in animals. Psychological Review, 43, 427-449.

Swartzentruber, D., \& Rescorla, R. A. (1994). Modulation of trained and extinguished stimuli by facilitators and inhibitors. Animal Learning \& Behavior, 22, 309-316.

(Manuscript received January 26, 2007; revision accepted for publication June 5, 2007.) 